\title{
Helen Salisbury: The gaps in our knowledge
}

\author{
Helen Salisbury GP \\ Oxford
}

At medical school we learn to recognise the patterns of symptoms and physical signs that add up to predefined diagnoses. They appear in the textbooks and again in exam scenarios, but many of the real patients we meet in the clinic don't fit neatly into diagnostic boxes. Sometimes they're just variants on a theme (the pneumonia without a cough, the painless heart attack), but a sizeable minority of patients in both primary and secondary care have symptoms we can't explain. Estimates vary, but this is said to be a feature of $20-50 \%$ of consultations in primary and secondary care. ${ }^{12}$

Patients typically want to know what's wrong, and they can be frustrated by the lack of answers. Doctors are made similarly uncomfortable by their inability to solve a patient's problem. In GP surgeries we frequently say, "I don't know exactly what's causing your symptoms, but I can reassure you that it isn't dangerous." Patients can leave feeling unsatisfied, especially if they don't think they've been believed or that their symptoms have been taken seriously. Doctors often come to dread consultations where they don't have an answer-where their medical toolkit, and by extension they themselves, are found wanting.

As medically unexplained symptoms are so common, it's important that we equip our students and junior doctors with the skills to navigate this territory without over-investigation and over-treatment. One of the difficulties is deciding when to teach this: students need to understand the possible explanations and to have a grasp of diagnostic reasoning before we confound them with what can't be explained.

There's also disagreement among doctors about exactly what we should be teaching. Some believe that, if no pathophysiological diagnosis can explain the symptoms, they are by definition a form of somatisation-a physical expression of psychological distress, even when that distress isn't apparent to the patient. On the other hand, there are doctors who, while admitting that somatisation is common, also believe that some symptoms are unexplained because our medical knowledge remains incomplete.

When I was a student in Whitechapel a very common complaint among some local women, who came originally from Bangladesh, was of pain "all over." This pain was medically unexplained, and theories about cultural difficulties in expressing psychological distress (which sometimes bordered on racist) were wheeled out to account for this somatisation. Since then we've gained a better understanding of vitamin D deficiency, which was likely to explain the pain experienced by some of these patients.

We need to review the learning needs of our students and juniors. But we also need to tackle the hubris of our profession and be able to say, "We don't know-yet."

\section{Competing interests: See www.bmj.com/about-bmj/freelance-contributors.}

Provenance and peer review: Commissioned; not externally peer reviewed.

1 Jadhakhan F, Lindner OC, Blakemore A, Guthrie E. Prevalence of medically unexplained symptoms in adults who are high users of health care services: a systematic review and meta-analysis protocol. BMJ Open 2019;9:e027922.

10.1136/bmjopen-2018-027922. 31270115

2 Chew-Graham CA, Heyland S, Kingstone T, etal . Medically unexplained symptoms: continuing challenges for primary care. Br J Gen Pract 2017;67:106-7. 10.3399/bjgp17X689473 28232334

Published by the BMJ Publishing Group Limited. For permission to use (where not already granted under a licence) please go to http://group.bmj.com/group/rights-licensing/ permissions 